\title{
Evaluation criteria for spectral design of camouflage
}

\author{
Christina Åkerlind, Jan Fagerström, Tomas Hallberg and Hans Kariis
}

\section{Linköping University Post Print}

\section{Tweet}

N.B.: When citing this work, cite the original article.

Original Publication:

Christina Åkerlind, Jan Fagerström, Tomas Hallberg and Hans Kariis, Evaluation criteria for spectral design of camouflage, 2015, Proc. SPIE 9653, Target and Background Signatures. http://dx.doi.org/10.1117/12.2194828

Copyright: SPIE

Postprint available at: Linköping University Electronic Press

http://urn.kb.se/resolve?urn=urn:nbn:se:liu:diva-123734 


\section{Evaluation Criteria for Spectral Design of Camouflage}

\section{Christina Åkerlind ${ }^{\mathrm{a}, \mathrm{b}}$, Jan Fagerström ${ }^{\mathrm{a}}$, Tomas Hallberg ${ }^{\mathrm{a}}$, Hans Kariis ${ }^{\mathrm{a}}$}

a) Division of Sensor and Electronic Warfare, Swedish Defence Research Agency (FOI), SE 58111

Linköping, Sweden, b) Department of Physics, Chemistry and Biology, Linköping University, SE 58183

Linköping, Sweden

\section{INTRODUCTION}

In development of visual (VIS) and infrared (IR) camouflage for signature management, the aim is to design surface properties of an object to spectrally match or adapt to a background and thereby minimizing the contrast perceived by a threatening sensor. The so called "ladder model" [1,2] (Fig. 1.) relates the requirements for task measure of effectiveness with surface structure properties through the steps signature effectiveness and object signature. It is intended to link materials properties via platform signature to military utility and vice versa. Spectral design [1] of a surface intends to give it a desired wavelength dependent optical response to fit a specific application of interest. Six evaluation criteria were stated [1], with the aim to aid the process to put requirement on camouflage and for evaluation. The six criteria correspond to properties such as reflectance, gloss, emissivity, and degree of polarization as well as dynamic properties, and broadband or multi spectral properties. These criteria have previously been exemplified on different kinds of materials and investigated separately [2]. Andersson and Åkerlind [1] further point out that the six criteria rarely were considered or described all together in one and same publication previously. The specific level of requirement of the different properties must be specified individually for each specific situations and environment to minimize the contrast between target and a background. The criteria or properties are not totally independent of one another. How they are correlated is part of the theme of this paper. However, prioritization has been made due to the limit of space. Therefore all of the interconnections between the six criteria will not be considered in the work of this report. The ladder step previous to digging into the different material composition possibilities and choice of suitable materials and structures (not covered here), includes the object signature and decision of what the spectral response should be, when intended for a specific environment. The chosen spectral response should give a low detection probability (DP). How detection probability connect to image analysis tools and implementation of the six criteria is part of this work.

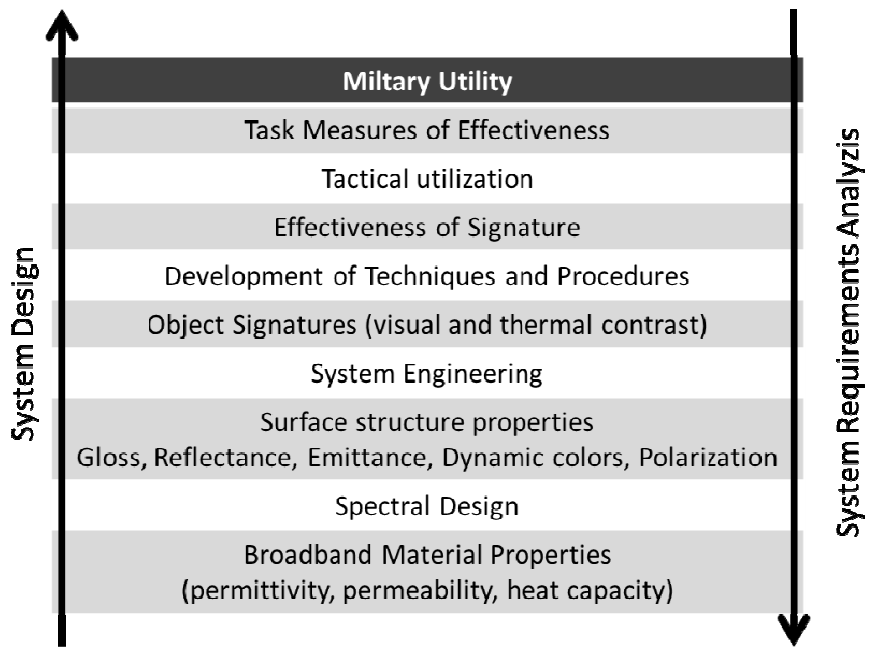

Figure 1. The so called "ladder model" links materials properties to military utility via several steps going both upwards and downwards using system design and system requirements analysis, respectively.

Target and Background Signatures, edited by Karin U. Stein, Ric H. M. A. Schleijpen, Proc. of SPIE Vol. 9653, 965303 (C) 2015 SPIE · CCC code: $0277-786 X / 15 / \$ 18 \cdot$ doi: $10.1117 / 12.2194828$ 
Dynamic (time dependent) properties are often the most revealing, at least to the human eye. The dynamics can be due to a background that changes due to, e. g., time of day, seasonal variations, weather or hostile activities, a moving target, or a target with changing optical properties. Dynamic properties could include a change of the reflectance (color) or emittance. Tuning the levels of the reflectance for a specific wavelength will realize dynamic properties [2] or controllability at optical wavelengths [1] of the spectral reflectance appearance over time, a property of switchable or adaptive properties. Often such a dynamic process involve different internal processes that deal with the material and structure composition. There are materials with dynamic properties in themselves, but often also the underlying structure for example electronic circuits or such has to be considered to a greater extent for realization. A dynamic process might also cause unintended change also to properties in other wavelength bands. Dynamic properties are not discussed further.

Examples of spectral bands of interest are VIS (400-800 nm), near infrared (NIR) (800-1200 nm), short wave IR (SWIR) (1.5-3 $\mu \mathrm{m})$, mid wave thermal IR (MWIR) (3-5 $\mu \mathrm{m})$ and long wave thermal IR (LWIR) (8-12 $\mu \mathrm{m})$. Radar frequencies are not included in the spectral range, but electric and magnetic material properties in these frequencies are still important issues and belong to the criterium broadband/ multispectral properties [2] or non-destructing properties [1]. The electric properties of radar absorption material might have interfering effect in the spectral range. In Table 1 examples of some for camouflage important material classes are listed with characteristic broadband properties. For this paper VIS, NIR and LWIR were chosen for further studies.

Table 1. Interesting material parameters in a broad band camouflage context.
\begin{tabular}{|l|l|l|l|l|}
\hline $\begin{array}{l}\text { Material } \\
\text { class }\end{array}$ & VIS property & NIR property & $\begin{array}{l}\text { LWIR } \\
\text { property }\end{array}$ & $\begin{array}{l}\text { Electric } \\
\text { property/radar }\end{array}$ \\
\hline Metal & Reflective & Reflective & Reflective & Conductive \\
\hline $\begin{array}{l}\text { Vegetation } \\
\text { (chlorophyll) }\end{array}$ & $\begin{array}{l}\text { Green, } \mathrm{H}_{2} \mathrm{O} \text { content, } \\
\text { Pigment dependent }\end{array}$ & Reflective & Emissive & Low conductivity \\
\hline Polymer & Pigment depending & Pigment depending & Emissive & Insulating \\
\hline Ceramic & Pigment depending & Pigment depending & Emissive & Insulating \\
\hline Semiconductor & Opaque (usually) & Opaque (usually) & Transparent & Semiconducting \\
\hline
\end{tabular}

In the design and evaluation phase the reflectivity properties in VIS, IR and for radar frequencies must be treated simultaneously. Although the material composition and its condition are relevant for the results, these aspects are not further discussed here.

Both emissivity, gloss and polarization properties are tightly connected to reflectivity. Besides angle and wavelength dependence, they are also sensitive to the orientation of the electrical field and the geometry of a scene. As man-made surfaces often are more glossy than natural objects, their polarization differ from the low polarized natural background. As technique and polarization sensitive sensor systems matures, polarization data will likely be more commonly used [3]. Forssell and Hedborg Karlsson report close to Lambertian radiation and depolarization properties for polarization studies performed on different surface roughnesses [4]. Forssell et al. report on detection of polarization [5].

There are a few examples of implementation of polarization aspects into analyze tools with different levels of readiness for use $[3,6,7]$. Polarization studies demand specialized analysis and computing tools, which is an explanation of why a systematic discussion of the impact of polarization is not included in this work. In this work we will focus on reflectance, gloss and emittance.

Reflectivity as a function of wavelength relates the interaction between light and material and the material parameters $\mathrm{n}$ and $\mathrm{k}$ through the Fresnell function [8]. Spectral directional hemispherical reflectance measurement data, attained from a spectrometer equipped with an integrating sphere, describes the relation between the irradiance of light incident from a specific direction and the total reflected radiation from a 
surface. This kind of data can be used to calculate the $C I E L^{*} a^{*} b^{*}$ values of the 3D-color space determined by the International Commission on Illumination [9], or other colour coordinate systems such as RGB or HSV which is used here [10].

Gloss can be expressed as angular dependent specular reflectivity. Requirements of gloss is often set for military equipment being purchased, and often the complex property of surface scattering is reduced to a single number in a gloss measurement which is a simplification of the gloss phenomena. In scientific contexts often the more complex bidirectional reflection distribution function (BRDF) is used. BRDF, describes the reflectance at the incoming angles $\left(\theta_{i}, \phi_{i}\right)$ and outgoing angles $\left(\theta_{r}, \phi_{r}\right)$, as a ratio between the scattered radiance $\left(I_{r}\right)$ and the incident irradiance $\left(I_{i}\right)$. [11].

The emissivity $\varepsilon$ of a surface for non-transmitting materials is related to the reflectance $\mathrm{R}$ and absorbance $\mathrm{A}$ through the relation $\varepsilon(\lambda)=A(\lambda)=1-R(\lambda)[12,13]$. Emitted light might, depending on the geometry and surface properties of the target, be polarized. [5]

A good way to quantify the detectability is to let a statistically relevant number of human observers look at the target in the background and determine the detection distance or time to detection. As this method requires huge resources and time, software based evaluation methods have been developed. In this work one such software package (Terrtex) [14] developed by FOI and BAE systems is used. Signature, features that separate the target from the background, is in Terrtex, quantified using the generalized signal to noise ratio function (GSNR) [15]. GSNR includes, not only the contrast between the target and the background but also features like edge concentration, curvature and spatial frequency strength. GSNR can be related to the detection probability or detection range [15]. These are in turn related to of camouflage effectiveness. In this work the GSNR function is sufficient to give values for a relative, but not absolute, comparison between surfaces. In this work we compare the GSNR of standard surfaces, more or less suitable, to study the effects of the parameters reflectance, gloss, temperature and emittance in selected wavelength bands well spread over the electromagnetic spectrum.

\section{METHOD AND EXPERIMENTAL DATA}

The method used rely on two simulation softwares (Terrtex and RadThermIR). The target chosen in the examples is called CUBI $[16,17]$ which also exist as a real object. Generating a simple case for exemplification, the CUBI was placed on a grass area not to get lost in complicated or classified backgrounds.

On the target, different properties were applied to illustrate variation in reflectance, gloss and emissivity values in different wavelength regions. Therefore separate images of CUBI were made for VIS, NIR and LWIR. Also different weather conditions were used in theses wavelengths regions.

In the VIS and NIR ranges a Swedish grass area on a cloudy summer day was chosen as background setting. Pictures of the area were recorded with visible, near infrared and ultra violet filters as well as with a thermal infrared camera. Color was applied on the sides of CUBI images and these were pasted in a photo of grass. Color (hue (H), saturation (S) and whiteness value (V)) in VIS, and grayscales (W\% ) in NIR were varied.

In LWIR the RadThermIR(C software (Thermoanalytics) was used to generate thermal images of the model object in a generic background (grass area). The settings were as for CUBI [17] i.e. the weather used was a sunny day October 21th 2010 at noon in Linköping, Sweden. And the graphic view was: azimuth -130 , elevation 30, roll: 0 , zoom 400, no offset, scale radiance 0-60.

BRDF depends on both the composition of the surface material and on the surface conditions and roughness. In the RadThermIR software, the BRDF model implemented is based on the Sandford-Robertson BRDF 
empiric model based on reflectance and polarizations data from large amount of aircraft paint [18]. In this model, the BRDF is described using four parameters: gazing angle, specular lobe width, diffuse reflectance factor and total hemispherical emissivity, where the two first are constants and the last two are functions of wavelength. The directional reflectance information is needed to determine the thermal emittance, solar absorptance- and thermal balance calculations.

In LWIR, models of real surface coating materials from the RadThermIR database were used to exemplify different emissivity values, $\varepsilon$. For some LWIR images, CUBI was heated by applying a $1500 \mathrm{~W}$ heater inside to get a variation in target temperature.

Synthetic images were produced for further analysis in Terrtex. Figure 2 shows the CUBI object, and Table 2 describes the different surface properties applied to the CUBI images.

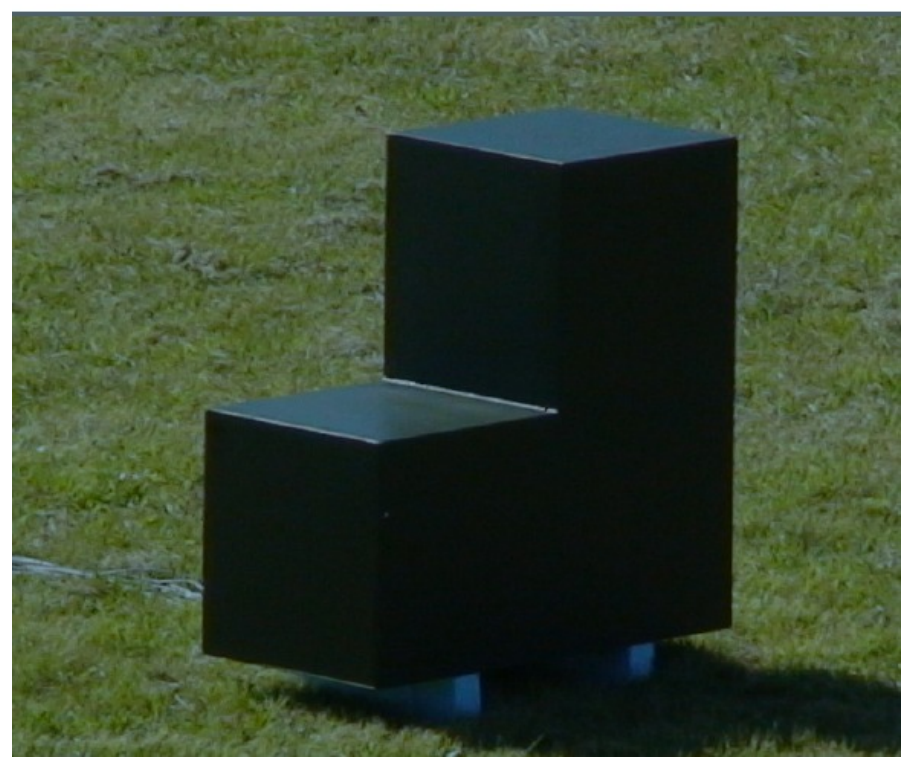

Figure 2. The generic ground object CUBI.

Table 2. Examples of alternated parameters of the surface properties applied to the CUBI in the different wavelength bands. The hue (H), saturation (S) and lightness $(V)$ were varied one at a time, while the other were kept constant.

\begin{tabular}{|l|l|l|}
\hline VIS & NIR & $\begin{array}{l}\text { LWIR } \\
\text { (All with 0 and } 1500 \mathrm{~W} \text { internal } \\
\text { heating.) }\end{array}$ \\
\hline $\mathrm{H} \pm 1, \mathrm{~S}, \mathrm{~V}$ & Black & Glossy metal \\
\hline $\mathrm{H}, \mathrm{S} \pm 1, \mathrm{~V}$ & Grey W 40\% & Matte metal \\
\hline $\mathrm{H}, \mathrm{S}, \mathrm{V} \pm 1$ & Grey W 50\% & Polyuretan paint \\
\hline & Grey W 60\% & Arbitrary paint, grey/green \\
\hline & White & Blackbody \\
\hline
\end{tabular}

The Terrtex software (FOI, BAE systems) was used to perform image analysis. The software takes an image as an input and calculates GSNR, which is related to detection probability for a target in a background. The Terrtex software version from 2011-11-10 for 64 bit processor was used. Grayscale (24 bit) bitmap images 
with a size of $640 \mathrm{x} 427$ pixels were imported into Terrtex. A mask was created to mark the target (CUBI) and an equally sized mask was put on a typical grass background.

The parameters listed in Table 3 were calculated and used to determine the GSNR the different wavelength regions:

Table 3. Properties used to determine the GSNR.
\begin{tabular}{|l|l|l|}
\hline & $\begin{array}{l}\text { Texture properties calculated and } \\
\text { used to determine the GSNR in VIS, } \\
\text { NIR and LWIR: }\end{array}$ & $\begin{array}{l}\text { In VIS also the colour dependent parameter } \\
\text { Edges was used. For the Gabor transform the } \\
\text { following values were used: }\end{array}$ \\
\hline 1 & Mean value of intensity & 6 frequencies \\
\hline 2 & Deviation within the region & 8 orientations \\
\hline 3 & Edge concentration & Window size 32 \\
\hline 4 & Laplace blob strength & Sigma 4.0 \\
\hline 5 & Gabor medium frequency band & Lower frequency: 0.12 \\
\hline 6 & Gabor high frequency band & Upper frequency: 0.25 \\
\hline 7 & Gabor energy & \\
\hline 8 & Gabor shape & \\
\hline 9 & Mean curvature & \\
\hline
\end{tabular}

Sensitivity analysis was employed to determine the influence of the criteria on the detection probability.

The following criteria were used:

- VIS reflectivity (parametrized into hue, saturation and whiteness)

- NIR reflectivity (gray scale)

- Gloss (in infrared generalized as BRDF) - glossy and matte Al surface

- LWIR emissivity $(8-12 \mu \mathrm{m})$, glossy and matte Al surface, blackbody and different paints

- CUBI temperature, varied by applying a heat source inside CUBI.

The change in GSNR with regard to the variation in each criterium was calculated. Table 4 below describe database parameters for the paints used in the LWIR case.

Table 4. Properties of the surfaces in LWIR.

\begin{tabular}{|l|l|l|l|l|l|l|}
\hline Nr & Surface & Description & $\begin{array}{l}\text { Solar } \\
\text { absorptivity }\end{array}$ & $\begin{array}{l}\text { Thermal } \\
\text { emissivity }\end{array}$ & $\begin{array}{l}\text { Fresnel } \\
\text { coefficient }\end{array}$ & $\begin{array}{l}\text { Specular } \\
\text { Lobe Width }\end{array}$ \\
\hline 2 & $\begin{array}{l}\text { Arbitrary } \\
\text { Green }\end{array}$ & $\begin{array}{l}\text { Green olive on } \\
\text { army vehicle }\end{array}$ & 0.89 & 0.90 & 0.12 & 0.5 \\
\hline 3 & $\begin{array}{l}\text { Arbitrary } \\
\text { paint, }\end{array}$ & $\begin{array}{l}\text { Gray weathered } \\
\text { paint on metal }\end{array}$ & 0.46 & 0.49 & 1.00 & 0.36 \\
\hline 4 & $\begin{array}{l}\text { Lusterless } \\
\text { paint }\end{array}$ & $\begin{array}{l}\text { Gray Polyuretan } \\
\text { paint, lusterless }\end{array}$ & 0.74 & 0.80 & 0.32 & 0.44 \\
\hline 6 & Glossy metal & bare polished Al & 0.25 & 0.026 & 0.001 & 0.003 \\
\hline 7 & Matte metal & $\begin{array}{l}\text { bare matte oxidized } \\
\text { Al }\end{array}$ & 0.52 & 0.16 & 0.092 & 0.028 \\
\hline 8 & Blackbody & $\begin{array}{l}\text { Black paint, low } \\
\text { reflectance }\end{array}$ & 0.94 & 0.92 & 0.057 & 0.45 \\
\hline
\end{tabular}




\section{RESULTS}

VIS

In the visible range, images of CUBI are synthetically put into a real scene of grass and small trees. Colour, as perceived by the human eye, can be described by three coordinates. For displays the RGB (red, green, blue) system is often used. We have chosen to do the analysis in the HSV system, where the appearance of a paint is described with the three parameters hue (an angle in the colour circle from where red is 0 , yellow 60, green 120 , blue 240), saturation (going from 0, which is gray, to 100, a fully saturated clear colour) and whiteness value (going from black, 0 to full lightness, 100), as in Fig. 3. HSV is a transformation of RGB into more intuitive coordinates.

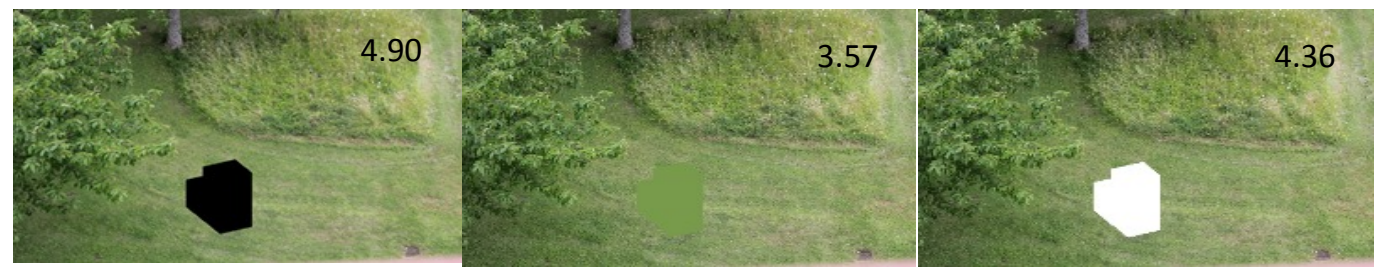

Figure 3. Example of whiteness value (going from black, 0 to full lightness, 100) combined with the obtained GSNR values.

$\mathrm{H}, \mathrm{S}$ and $\mathrm{V}$ were varied independently with pictures generated of CUBI with synthetic paints placed on the real grass surface. The images are analyzed with the Terrtex software, generating a GSNR value for each image. GSNR values plotted against the HSV-values is used to determine how sensitive the detection probability is to a change in each value.

As can be seen from Figures 3-6, and also deducted from the Terrtex analysis, the level of whiteness has large impact on the detection probability, whereas the influence of the hue is smaller and the saturation is almost neglectable.

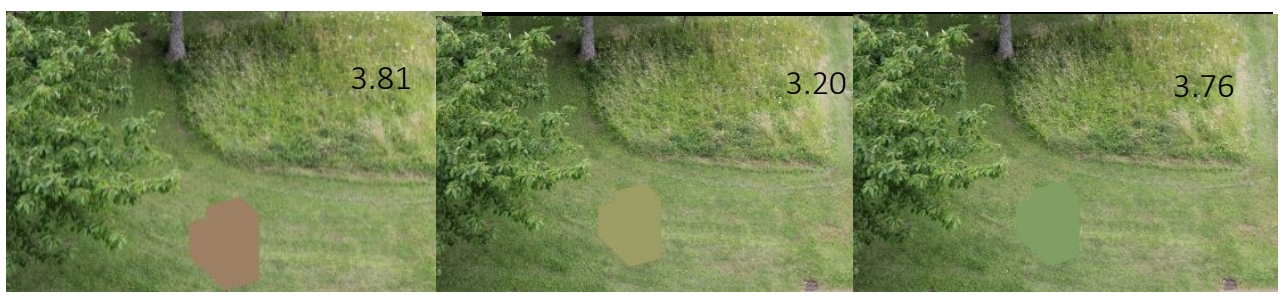

Figure 4. Synthetic CUBI on grass. The saturation of the CUBI-colour is $36 \%$ and the whiteness value is 62 in all cases. The hue is 30, 61 and 90 respectively.

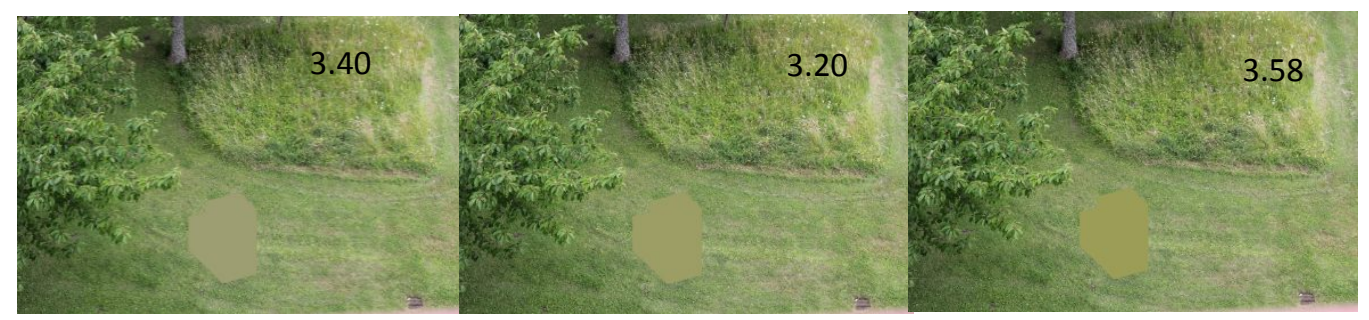

Figure 5. Synthetic CUBI on grass. The hue corresponds to the average hue of the grass background (60) and the whiteness value is 62 in all cases. The saturation of the CUBI-colour is, from left to right, 28\%, 36\% and 44\%. 


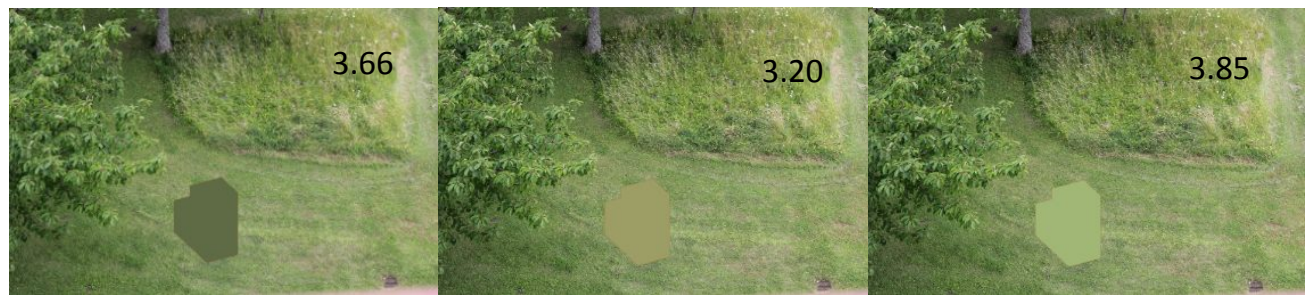

Figure 6. Synthetic CUBI on grass. The hue corresponds to the average hue of the grass ackground (61) and the saturation is $36 \%$ in all cases. The whiteness value is 42, 62 and 72, respectively.

\section{NIR}

The GSNR as a function of whiteness in NIR is illustrated in Fig. 7. Corresponding NIR images is shown in Fig. 8.

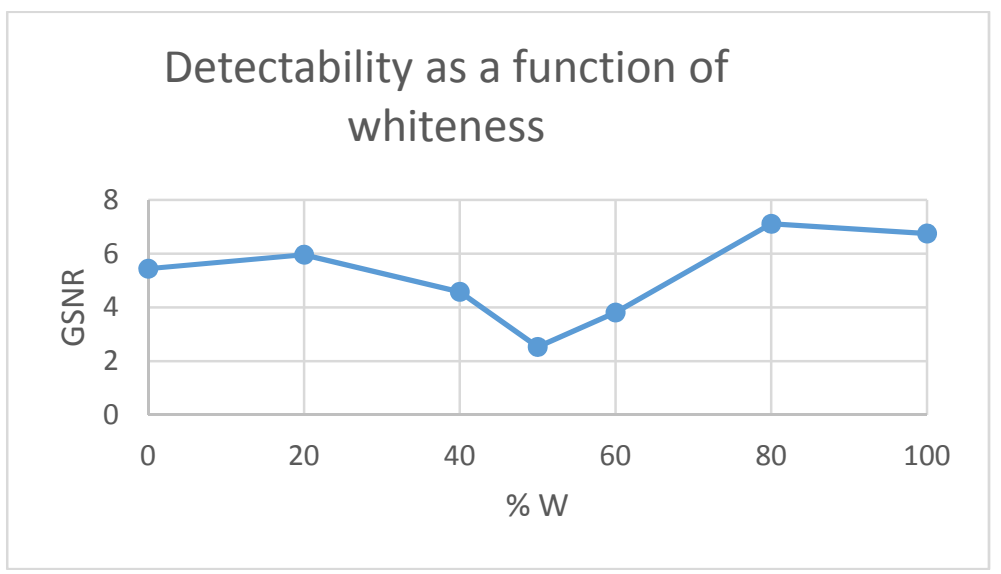

Figure 7. The GSNR as a function of whiteness. Texture features used: Mean, Local dev, Edge conc., Laplace, Gabor: low, med, high, energy, shape, Mean curv., 6 freq. 8 orientations.
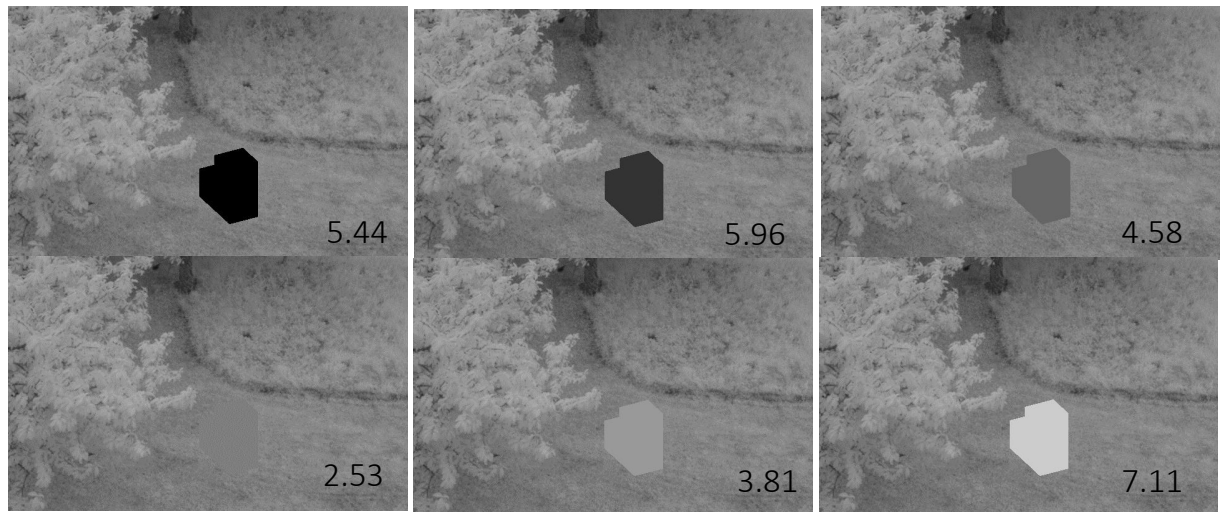

Figure 8. The effect of whiteness (from left to right: 0, 20, 40, 50, 60 and 80\%) in the NIR case is shown together with the GSNR values. 


\section{LWIR}

In the thermal infrared region $(8-12 \mu \mathrm{m})$ images were generated of CUBI with different surface coatings placed on a synthetic grass surface. Figure 9. shows the different surfaces in the LWIR analysis.
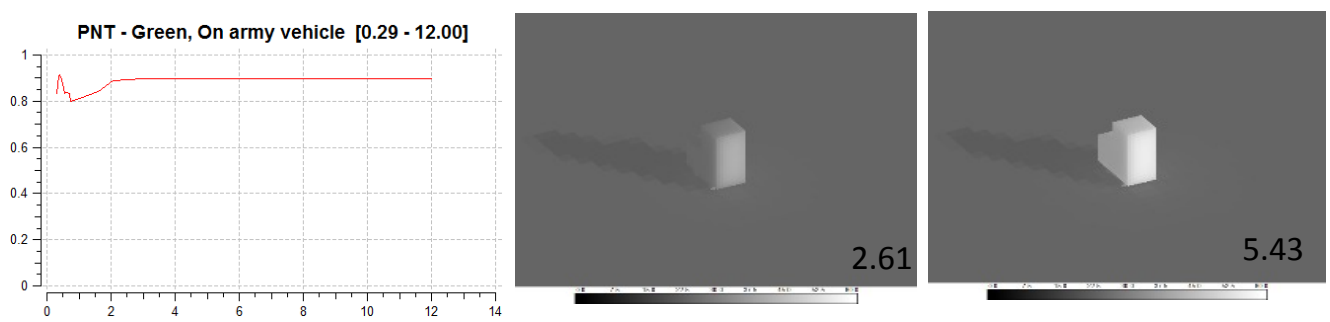

PNT - Gray, Weathered sl chipped, On metal (NEF0781) [0.30 - 14.10]
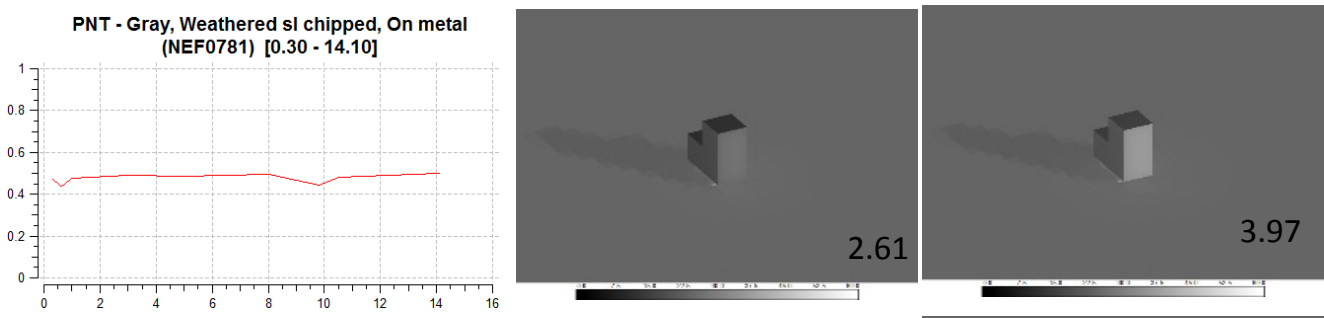

PNT - Gray, Polyurethane lusterless, On AI (NEF0743) $[0.30-12.60]$
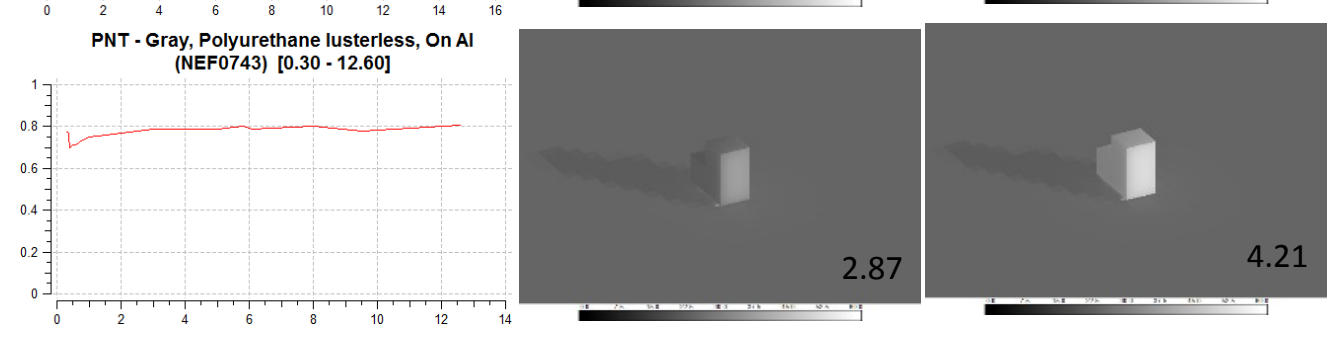

MTL - Aluminum, Bare polished (NEF0539) [0.30 12.60]
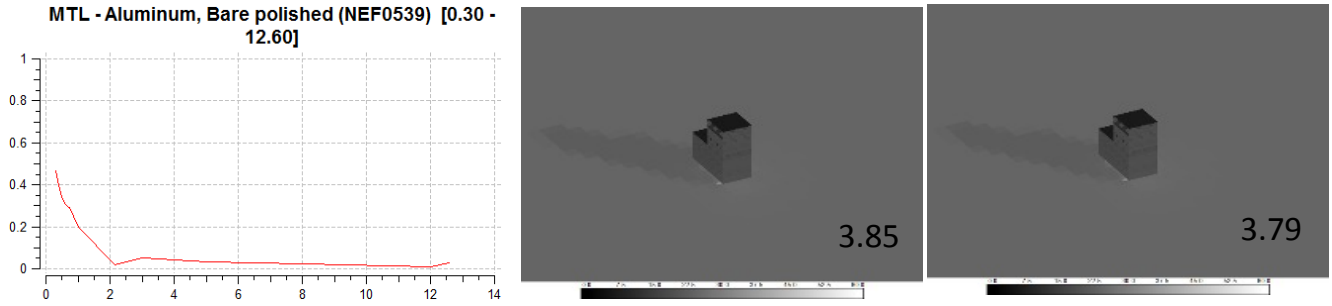

MTL - Aluminum, Bare, Matte oxidized plate (NEF0014) [0.42 - 14.10]
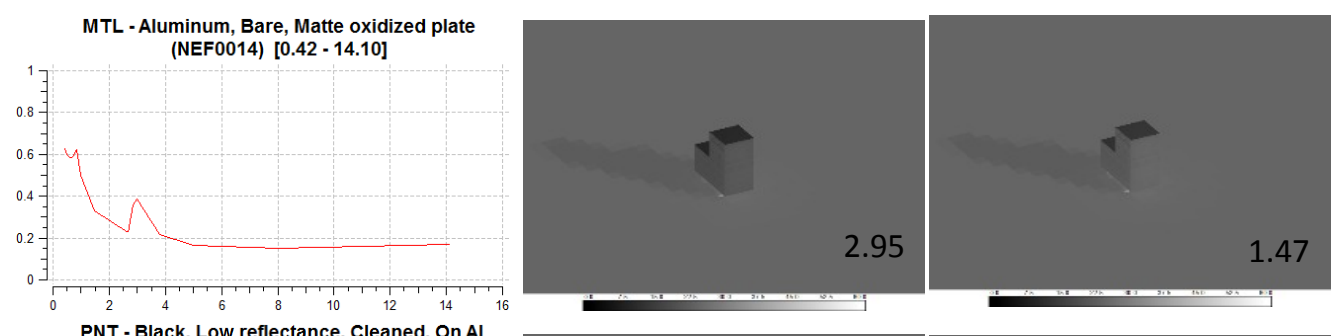

PNT - Black, Low reflectance, Cleaned, On AI (NEF0772) $[0.30-12.60]$
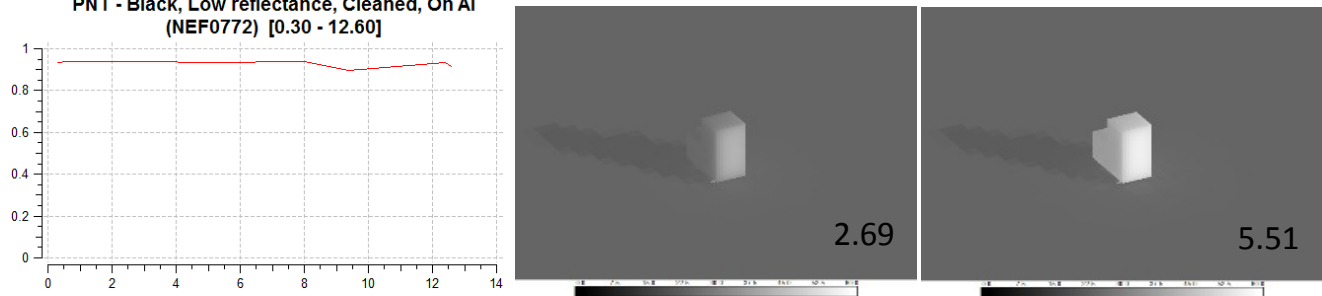

Figure 9. The different surfaces in the LWIR case. Columns from left to right: emissivity curve as a function of wavelength (from paint database); Image of the generated radiation in 8-12 $\mu \mathrm{m}$ for CUBI with internal heating $(0 \mathrm{~W}$, middle) and $(1500 \mathrm{~W}$, right) with the different coatings. 
Table 5 shows the GSNR results for the different surfaces.

Table 5. Used texture features were: Mean, Local dev, Edge conc., Laplace, Gabor: low, med, high, energy, shape, Mean curv., 6 freq. 8 orientations.

\begin{tabular}{|l|l|l|l|}
\hline $\mathbf{N r}$ & Description & GSNR $(0 \mathrm{~W})$ & GSNR $(1500 \mathrm{~W})$ \\
\hline 1 & Blackbody & 2.69 & 5.51 \\
\hline 2 & Green paint & 2.61 & 5.43 \\
\hline 3 & Gray paint & 2.61 & 3.97 \\
\hline 4 & Gray PU lusterless & 2.87 & 4.21 \\
\hline 6 & Polished Al & 3.85 & 3.79 \\
\hline 7 & Matte Al & 2.95 & 1.47 \\
\hline
\end{tabular}

The low emissive aluminum has high reflection. In this geometry, the vertical surfaces reflect the grass surface whereas the horizontal top surface reflect the cold sky. Low emissive CUBI does not exhibit much difference when heated (right).

The CUBI was internally heated to simulate a vehicle with an internal heat source (i. e. engine). For a high emissive paint the difference between the heated and unheated CUBIs is large. For these CUBIs the infrared images show essentially the actual surface temperature with a neglectable background reflection component.

Note that the actual surface temperature can be higher for a heated CUBI with reflective surface than for the highly emissive surface because less energy can be radiated away impeding the cooling of CUBI.

A CUBI highly reflecting in the visible and near infrared wavelength ranges absorb less sunlight than a CUBI with absorbing surface.

\section{DISCUSSION AND FUTURE WORK}

In the procurement and the included requirement setting process, reviewed by Andersson [19], measures of relevance for the application and field of use is important. The six criteria discussed in the introduction, were thought to be usable through the requirement setting and evaluation process aided by the so called "ladder model" (Fig.1) [1]. It is here found that, in some phase related to evaluation and the system design, other aspects and parameters rather than the six criteria are dimensioning. Therefore the interfaces between the steps in the "ladder model" has to be further elaborated. The physics parameters in the lower steps are not compatible with the image analysis and computer modelling parameters in the middle steps, which, in turn, are not compatible with the military parameters in the top steps.

As an example, overall reflectance is not the only relevant property for VIS properties in an analyzing software. Instead factors such as color, hue, saturation and whiteness are of importance of how an image is appearing and viewed. Also properties such as perception and statistical variations play an important role. Here we find that the impact of GSNR from whiteness is higher than for hue and saturation.

It is reported [20] that properties such as gloss, translucency and texture are also effecting the perception of a color of an object. As texture deeply effects properties such as gloss it is suggested to establish a "standard" texture to future characterization. Farrier [21] has studied the relation between gloss, roughness and BRDF for camouflage paints and questions the use of gloss as a requirement criteria. She suggests roughness properties as a substitute to a specified gloss as a dimensioning requirement.

In NIR a grayscale alone is used. To our knowledge no other variable parameters are relevant for this range, when the sensor is assumed to be a night vison goggle. It is however known that matching the chlorophyll curve is a challenge for this region when dealing with the spectrally resolved sensors, which are likely to impose a threat in the future. Also, different foliage have different properties. A new aspect could be to add a 
chlorophyll lookalike factor. This aspect is however not straight forward to solve, and is saved for future work.

In LWIR emissivity and temperature play a huge role. The difference in target and background temperatures have to be considered, i.e. if the target has higher temperature than the background it should have lower emissivity in order to minimize the contrast. Thereby also the detectability is minimized which is the aim of camouflage and signature management technology. A cold target with low emissivity or a low emissive surface oriented to give a reflection of the sky can give a negative contrast, reveling the target.

\section{Broad band}

Diffuse reflectance and low gloss are related and can be achieved through surface roughness. Both reflected and emitted radiation have polarization properties, which can be affected by the surface properties. These properties are also wavelength dependent, and must be viewed in a broad band perspective. From a spectral point of view, the surface and bulk scattering properties, as well as the wavelength dependent penetration depth are of relevance for surface appearance. For example, for radar frequencies a painted metal surface usually have metallic surface properties. In the visible however, the glossy metal is no longer the surface, the paint is. Another example of size dependence is that a visually matte surface, with micrometer size roughness structures, can be specularly reflecting in LWIR where the roughness $<<\lambda$. In the visible range, spectral reflectance is correlated to perceived color, whereas the reflectance in the thermal infrared range most often is given as an average over the sensor sensitivity range. Hyperspectral sensors with spectral sensitivity impose a future threat.

\section{Emissivity and BRDF}

In the used software RadThermIR, the angle dependence of radiance in terms of BRDF was incorporated in the rendered images using the Sandford-Robertson model. This model connects the angle dependence and the emittance associated it with a specular lobe width which is here a constant $[18,22]$. The relation between BRDF and emittance in this model is not straight forward. Synthetic paints with controlled roughness properties, for systematic studies, were not available for this study.

\section{Gloss and BRDF}

The use of gloss as a criteria of requirement of camouflage paint is questioned by Farrier [21]. Often the requirements of gloss are put rather low for matte paint, which instead gives problems with durability of the paint. A low gloss surface is often difficult to keep clean. Hence, the gloss requirements should not be stricter than necessary. Farrier further suggests BRDF, to be a better measure than gloss and that the BRDF angle should to be specified and associated with certain threat conditions [21]. On the other hand are gloss measurements easier to perform. It is a mobile technique that is convenient to measure also in the field compared to the complex BRDF measurements. If BRDF parametrization is to be used for future quantitative evaluation of surfaces, more effort has to be put in to this theme [23]. The BRDF width and height of the specular lobe is directly related to the surface topography or the roughness which makes the technique sensitive to different kinds of roughness. A problem using BRDF is to analyze the data and understand the additional information, compared to a gloss measurement [24]. Another disadvantage with using BRDF is that it requires a complicated measurement setup only available at a few laboratories. Arney et al. [24] confirm a correlation between the gloss index and the BRDF width, governed by the sample roughness. The BRDF technique enable the distinction between specular reflection, surface roughness and subsurface effects (bulk scatter). IR studies at FOI show a similar relationship between the degree of diffuse reflectance (DoDR) and the full width at half maximum (FWHM) of the BRDF peak (including specular and diffuse scattering). The 
DoDR is in this case measured with an integrating sphere for which the specular reflected light is removed via a port plug in the wall of the integrating sphere.

\section{Polarization and BRDF}

Polarized light is surface sensitive, partly due to aspects of the Brewster angle. At this angle the s-polarized light is the dominating part of the reflected light as the p-polarized part is more or less absorbed, depending on the surface properties. Studies on scattering and polarization properties of rough surfaces are suggested [25].

An early modeling of polarization in a scene has been performed [26]. Forssell claimes the importance of measuring different angles of incident and reflected radiance, especially for models of objects in a background. Meyers [3] has implemented modelling further and tried to model polarimetric BRDF, but the lack of BRDF data of a wide range of materials slows down the development and usage of polarized and unpolarized BRDF modelling. The fact that the optical parameters $\mathrm{n}$ and $\mathrm{k}$ can be derived empirically from pseudo-BRDF, according to an ARFL work, Meyers declares [3] makes things a little easier. Another example is Renhorn who has worked on implementation of a 4-parameter model regarding the p-BRDF [7]. Meyer [3] shows a connection between the roughness parameter and the BRDF lobe. An increased roughness gives a decreased height and increase in width of the BRDF curves, similar to Areny [24]. Also small variations in the surface roughness show vast effects of the nature of the predicted BRDF in specular aspects [3].

\section{GSNR}

With the synthetically generated hyperspectral images for remote sensing analysis in mind, synthetic images are more cost efficient to produce than real collected images. Identical scenes can easily be constructed for comparing different conditions, keeping all independent variables constant, thereby idealizing the case. The generalized signal to noise ratio (GSNR) gives a number of the importance of the different properties related to the identified important criteria: reflectance in VIS (hue, saturation and whiteness), NIR (shade of grey), and LWIR (emissivity, temperature).

The GSNR was found to be a suitable value when discussing properties of requirement for evaluation as it is linked to the, for applications relevant, detection probability (DP), DP=1-exp -( $\left.k^{*} \mathrm{GSNR}\right)$, where $\mathrm{k}$ is an observer dependent constant [15]. To express the GSNR into DP, observer statistics must be implemented. In our work however the value has not been converted to DP, since we are not studying sensor and observer aspects that would be needed in the model in order to get real DP. The GSNR value can, however, be efficiently used for relative comparison between different targets under the same conditions.

In our image analysis and extraction of GSNR values, the gloss parameter was not used. To extract gloss right from images involves an advanced photo-geometric analysis and 3D surface geometry [27] which was not the intention here. We should still remember that glossy surfaces can reveal an object with perfectly matched colour and thermal emission, especially if oriented in such a way that specular sun reflexes reach the observer.

\section{Future work}

Future work will include polarization impacting surfaces, both polarizing and depolarizing. It is needed to study how polarization changing surfaces are to be constructed for a wide wavelength range. It is needed to study the probability of a threat scenario of polarimetric sensors.

Motion, that is known to be very revealing, has not been included in this work. Dynamic properties will be at focus in future work, to understand more about the prominent detection factor related to change. 


\section{SUMMARY AND CONCLUSIONS}

This work can be seen as a continuation of the work presented by Andersson 2014. [1] Now, however, the starting point is in the middle of the "ladder model" (Fig 1). It does not aim at a complete analysis, but gives insight in the "ladder model" from another perspective.

When comparing and relating the six requirement dimensioning parameters of relevance for camouflage material, studies of the GSNR of different properties have been performed in VIS, NIR and LWIR using the RadThermIR and Terrtex software.

A number of conclusions could be drawn from the simulations, such as:

- Whiteness is a more important factor than hue and saturation for the detection range of our model target.

- Temperature is important for detectability of high emissivity surfaces, whereas the mirrored background is more important for low emissivity surfaces.

- The "ladder model" intended to combine the military utility with material parameters, using relevant criteria, was not sufficient. The physics parameters in the lower steps are not compatible with the image analysis and computer modelling parameters in the middle steps, which in turn, are not compatible with the military parameters in the top steps. Therefore the interfaces between the steps in the "ladder model" has to be further elaborated.

To achieve values relevant for evaluation of requirements in order to achieve a low signature or low contrast to the background the impact of roughness and texture need to be studied. Polarization and dynamic effects have to be further studied.

\section{ACKNOWLEDGEMENTS}

The Swedish Armed Forces are acknowledged for funding this work.

\section{REFERENCES}

[1] Kent E. Andersson, and Christina Åkerlind, "A review of materials for spectral design coatings in signature management applications," Proc. SPIE 84 92530Y, 1-20 (2014)

[2] Christina Åkerlind, “Optical Studies of Materials for Spectral Design,” Licentiate thesis at Linköping University , (2015)

[3] Jason P. Meyers, "Modeling Polarimetric Imaging using DIRSIG", A dissertation thesis at Chester F. Carlson Center for Imaging Science, Rochester Institute of Technology, (2002)

[4] Goeran Forssell, and Eva Hedborg-Karlsson, "Passive IR polarization measurements applied to rough surfaces consisting of cenosphere particles covered with gold," Proc. SPIE 4819, 145 (2002)

[5] Göran Forssell, and Eva Hedborg-Karlsson, "Measurements of polarization properties of camouflaged objects and of the denial of surfaces covered with cenospheres," Proc. SPIE Vol. 5075, 246 (2003)

[6] Ingmar G. E. Renhorn, Tomas Hallberg, David Bergström and Glenn D. Boreman, "Four-parameter model for polarization-resolved rough-surface BRDF,“ OPTICS EXPRESS 19, 1028 (2011)

[7] Ingmar G. E. Renhorn, "Efficient Polarimetric BRDF Model,” Renhorn-IR in manuscript 003 , (2015)

[8] E. Hecht, Optics, Addison-Wesley, (2002)

[9]"Colorimetry, 3d ed. draft CIE15.3:2004," CIE Technical Report, (2004)

[10] Noor A. Ibraheem, Mokhtar M. Hasan, Rafiqul Z. Khan, Pramod K. Mishra, "Understanding color Models: A

Review," ARPN Journal of Science And Technology.2, 265-275 (2012) 
[11] F. E. Nicodemus, "Reflectance nomenclature and directional reflectance and emissivity," Appl. Opt. 9, 1471-1475 (1970)

[12] SAT-handbok, Mark, ed. Lars Bohman, FOI ISBN 91-7056-114-1 (2003)

[13] J.M. Palmer. "The measurement of transmission, absorption, emission, and reflection", in: M. Bass,(Ed.). Handbook of Optics, volume II Devices, Measurements, and Properties. McGraw-Hill, Inc., (1995)

[14] Sten Nyberg, and Magnus Uppsäll, “TERRTEX - a software package for texture analysis,” FOA-R--97-00574-615 (1997)

[15] Sten Nyberg, "Models used in assessment of optical sensors," FOI-R--2290--SE, (2007)

[16] Jan Fagerström, Tomas Hallberg, Annica Hjelm, Nils Karlsson, Roland Lindell och Andras Persson, "IR-analys av ett generiskt markobjekt CUBI konstruktion, mätningar och simuleringar," FOI-R--2888--SE, (2009)

[17] Jan Fagerström, Annica Hjelm, Nils Karlsson, Roland Lindell och Jonas Rahm, ”Termiska beräkningar på två varianter av referensobjekt CUBI, Känslighetsanalys och jämförande mätningar,” FOI-R--3268--SE, (2011)

[18] Brian P. Sandford, and David C. Robertson, "Infrared reflectance properteis of aircraft paints," Technical report ESC-94-1004 (1994)

[19] Kent E. Andersson, Hans Kariis and Gunnar Hult, "A system approach to stealth on ground revisited," Proc. SPIE 9653, $1(2015)$

[20] CIE 175:2006 A Framework for the Measurement of Visual Appearance, ISBN 978390190652 7, (2006)

[21] Lisa M. Farrier, "Influence of surface roughness on the specular reflectance of low gloss coatings using bidirectional reflectance measurements" Thesis, AFRL-ML-WP-TM-2007-4019 (2006)

[22] RadThermIR Paint Creation Process, ThermoAnalytics Inc., Revision 1, 3/27/2012 (2012)

[23] James Jafolla, David Thomas and John Hilgers, "A Comparison of BRDF Representations and their Effect on Signatures," ADA399506, (1998)

[24] Jonathan S. Arney, Ling Ye, and Jiff Wible and Tom Oswald, "Analysis of Paper Gloss,” J. Pulp \& Paper Sci, $32 \mathrm{~N} 1,19-23$ (2006)

[25] Göran Forssell, "Passive optical polarisation applied on camouflage objects," FOI-R--0594-SE, (2002)

[26] Göran Forssell, "Model calculations of polarization scattering from 3-dimensional objects with rough surfaces in the IR wavelength region," Proc. of SPIE 588818, 1 (2005)

[27] Juno Kim, Barton L. Anderson, "Image statistics and the perception of surface gloss and lightness," Journal of Vision 10, 3 (2010) 